\title{
Development of squamous cell carcinoma in an untreated patient of psoriasis: a case report
}

\author{
Zahra Talebzadeh ${ }^{1}$, Mahsa Bahraminejad $^{2}$, Zahra Malakoutikhah $^{1}$, and ali asilian ${ }^{3}$ \\ ${ }^{1}$ Affiliation not available \\ ${ }^{2}$ Isfahan University of Medical Sciences \\ ${ }^{3}$ Skin Disease Research Center and Department of Dermatology, Isfahan University School \\ of Medicine, Isfaha, Iran
}

December 17, 2020

\begin{abstract}
In this article we report a case of SCC in an untreated 45-year-old male patient of psoriasis. The patient referred to the dermatology department manifesting erythrodermic psoriasis. After taking multiple biopsies from scalp lesions, the result was reported to be SCC with sebaceous differentiation.
\end{abstract}

\section{Introduction}

Psoriasis is a chronic, inflammatory, immune-mediated disease of the skin induced by excessive activation of adaptive immune system. Different cell types release cytokines which lead to activation of myeloid dendritic cells. Subsequently myeloid dendritic cells activate $\mathrm{T}_{\mathrm{H}} 1, \mathrm{~T}_{\mathrm{H}} 17$ and $\mathrm{T}_{\mathrm{H}} 22$ through interleukin-12 (IL-12) and IL-23 secretion. These $\mathrm{T}$ helper cells eventually lead to downstream proliferation of keratinocytes, overexpression of endothelial adhesion molecules, angiogenesis and infiltrations of immunocytes to the skin which are responsible for psoriasis development. ${ }^{1}$

\section{Case presentation}

In this case we report a 45-year-old patient with untreated psoriasis who referred to dermatology inpatient department due to erythrodermic psoriasis, through further evaluation several biopsy specimens were taken from patient and the result of scalp biopsy was reported to be SCC. Although this type of NMSC is prevalent among under treatment psoriatic patients, our case had not received any systemic therapy or phototherapy. The patient was a 45-year-old, mental retard male, referred to the dermatology inpatient department due to erythrodermic psoriasis. He was a smoker, unemployed and poor hygiene person who did not follow up his medical condition over the years. Through his admission, complete history was taken and it revealed that he was suffering from localized plaque-type psoriasis for 10 years and was only under treatment of topical corticosteroids including triamcinolone or clobetasol two or three times per day as well as topical calcitriol and emollients. The patient had no history of phototherapy or any oral antipsoriatic agents. Family history of psoriasis, skin cancers and other related disorders was also negative. Through admission the patient had low grade fever and malaise. On dermatological examination we found diffuse erythematous scaly eruptions over his scalp, trunk and bilateral extremities, involving $90 \%$ of the body surface area [Figure 1, 2]. They were associated with moderate to severe pruritus. A $6 \times 5 \mathrm{~cm}$ erythematous, firm, proliferative growth, adherent to the underlying structures was present over the frontal part of his scalp for 2 years. Furthermore multiple crusted tumoral lesions were existent diffusely on the scalp [Figure 3]. These lesions were not related to previous psoriatic plaques. No lymphadenopathy or mucosal involvement were detected. Nails pitting and onycholysis were seen in hand and toe nails. Other evaluations including complete blood 
cell count, electrocardiogram and brain CT scan were normal. Multiple skin biopsies were taken from his scalp and trunk, histopathological investigation of scalp specimen revealed neoplastic proliferation of epithelial cells with pushing border and surface ulceration in focal area and clear cell changes in some area. Neoplastic pleomorphism cells had vesiculated nuclei and marked mitotic figure was also seen. In another pieces tumoral bulk did not have attachment to epidermis [Figure 4]. IHC staining showed strongly positive P63, positive EMA and CK and negative Ber-EP4. These findings were more favor for squamous cell carcinoma with sebaceous differentiation. Trunk skin lesion biopsy findings were also consistent with psoriasis [Figure 5]. Eventually the largest tumoral lesion was removed through Mohs surgery. Other lesions treated with intralesional injection of interferon alfa-2a 1.5 million units twice a week.

\section{Discussion \& Conclusion}

Psoriasis is a systemic immune-mediated and genetic disorder defined by overgrowth and differentiation of keratinocytes. Environmental stimuli trigger the process of disease in susceptible individuals. This pathologic interaction occurs among skin cells, immunocytes and numerous biologic signaling molecules. Through activation of $\mathrm{T}_{\mathrm{H}} 1, \mathrm{~T}_{\mathrm{H}} 17, \mathrm{~T}_{\mathrm{H}} 22$ cells, these cells cause a chronic inflammatory state, alter proliferation of keratinocytes and release of several interleukins responsible for development of psoriasis. ${ }^{1}$ Increased risk of malignant cancer development in patients with psoriasis has been investigated recently. In several studies, the total cancer incidence rate was higher than general population. Subsequently SCC was the second most prevalent cancer among psoriatic patients. ${ }^{2}{ }^{3}$ However it is still unclear whether the correlation of SCC with psoriasis is due to underlying genetic basis of disease or its treatments. Phototherapy, immunosuppressive therapy and other well-known therapeutic methods have made it challenging to estimate the baseline risk of SCC incidence in psoriatic patients. So far several studies showed positive association of such therapies on development of SCC. And the effect of certain psoriasis treatments on incidence of SCC has been proven. ${ }^{4}$ A systematic literature review conducted by E. Archier et al. showed an increased risk of SCC following oral psoralen and ultraviolet A (PUVA), a considerable and advantageous therapeutic method. ${ }^{5}$ Systemic non-biologic therapies such as methotrexate and cyclosporine have also been associated with higher risk of malignancies. These two agents are associated with increased risk of SCC in psoriatic patients, elevated by PUVA therapy. Moreover tumor necrosis factor-a Inhibitors (anti-TNFa) were associated with considerably higher risk of SCC development in psoriatic patients, increased by primary PUVA exposure and/or immunosuppressive therapy. ${ }^{4}$ However since most studies include treated and untreated patients together, we have no available data on potential risk of SCC development in patients without any well-defined therapy. In this article we report a 45-year-old male patient with psoriasis who have been treated with topical agents and multiple tumoral lesions developed over his scalp eight years after his first initial diagnosis. This case states that the potential risk of SCC development in patient with psoriasis is probably not just due therapeutic side effects. Further investigations are needed to clarify underlying pathobiology of SCC development in these patients. Moreover periodic screening for prevalent cancers such as SCC is warranted in patients with psoriasis.

\section{List of abbreviations}

CK: cytokeratin

EMA: epithelial membrane antigen

IHC: immunohistochemistry

IL: interleukin

NMSC: non-melanoma skin cancer

PUVA: psoralen ultraviolet A

SCC: squamous cell carcinoma

$\mathbf{T}_{\mathbf{H}}: \mathrm{T}$ helper 
TNF-a: tumor necrosis factor-a

\section{Declarations}

Ethics approval and consent to participate

Not applicable

\section{Consent for publication}

"Written informed consent was obtained from the patient for publication of this case report and any accompanying images. A copy of the written consent is available for review by the Editor-in-Chief of this journal."

\section{Availability of data and materials}

The datasets generated and/or analysed during the current study are available in the [NAME] repository, [PERSISTENT WEB LINK TO DATASETS]

\section{Author contributors}

Not applicable

\section{Funding}

Not applicable

\section{Competing interests}

The authors declare that they have no competing interests.

\section{Acknowledgements}

Not applicable

\section{References}

1. Armstrong AW, Read C. Pathophysiology, Clinical Presentation, and Treatment of Psoriasis: A Review. Jama. 2020 May 19;323(19):1945-60.

2. Wang X, Liu Q, Wu L, Nie Z, Mei Z. Risk of non-melanoma skin cancer in patients with psoriasis: An updated evidence from systematic review with meta-analysis. Journal of Cancer. 2020;11(5):1047.

3. Hannuksela-Svahn A, Pukkala E, Läärä E, Poikolainen K, Karvonen J. Psoriasis, its treatment, and cancer in a cohort of Finnish patients. Journal of investigative dermatology. 2000 Mar 1;114(3):587-90.

4. Geller S, Xu H, Lebwohl M, Nardone B, Lacouture ME, Kheterpal M. Malignancy risk and recurrence with psoriasis and its treatments: a concise update. American journal of clinical dermatology. 2018 Jun $1 ; 19(3): 363-75$.

5. Archier E, Devaux S, Castela E, Gallini A, Aubin F, Le Maître M, Aractingi S, Bachelez H, Cribier B, Joly P, Jullien D. Carcinogenic risks of psoralen UV-A therapy and narrowband UV-B therapy in chronic plaque psoriasis: a systematic literature review. Journal of the European Academy of Dermatology and Venereology. 2012 May;26:22-31.

\section{Figures}




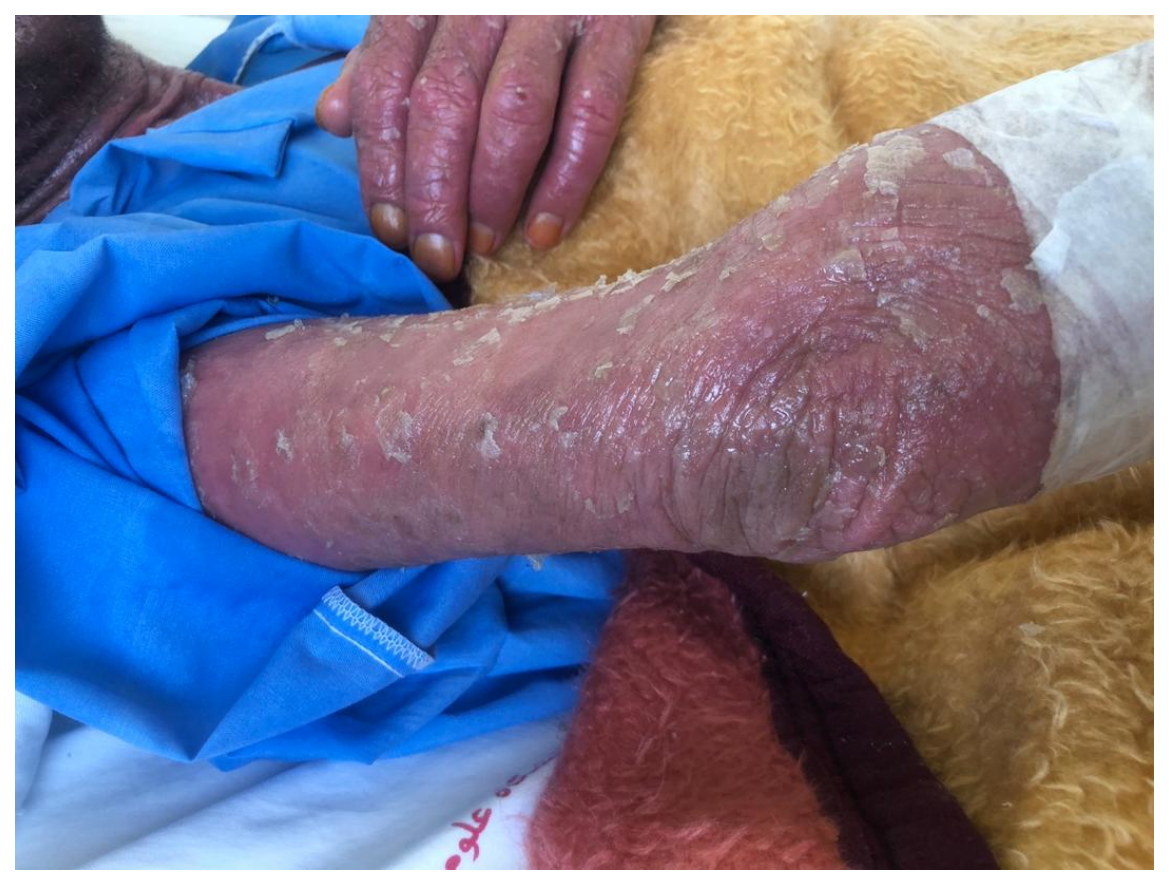

Figure 1: Extensor aspect of the right arm showing erythematous scaly skin. 


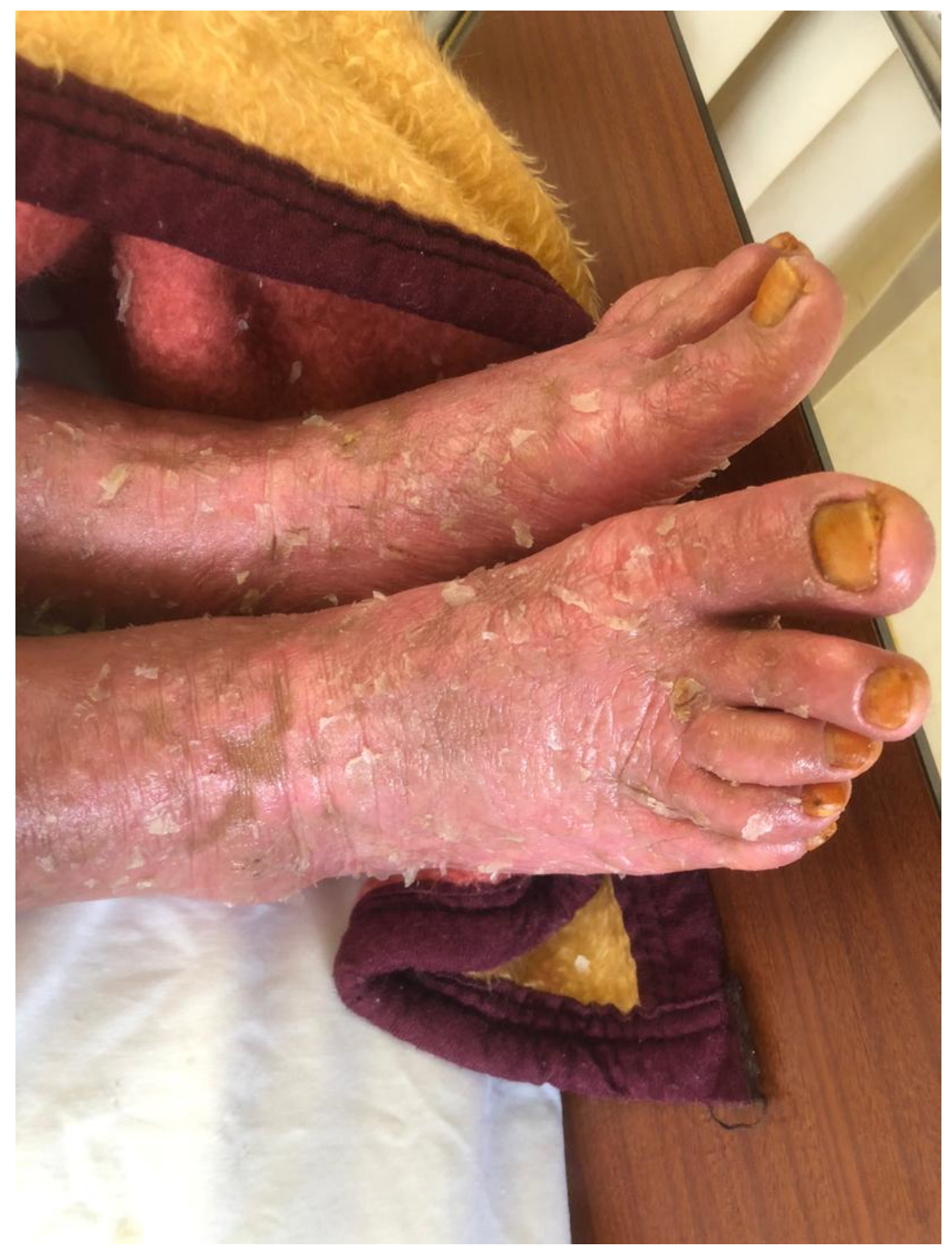

Figure 2: Dorsal aspect of both feet showing erythematous scaly skin. 


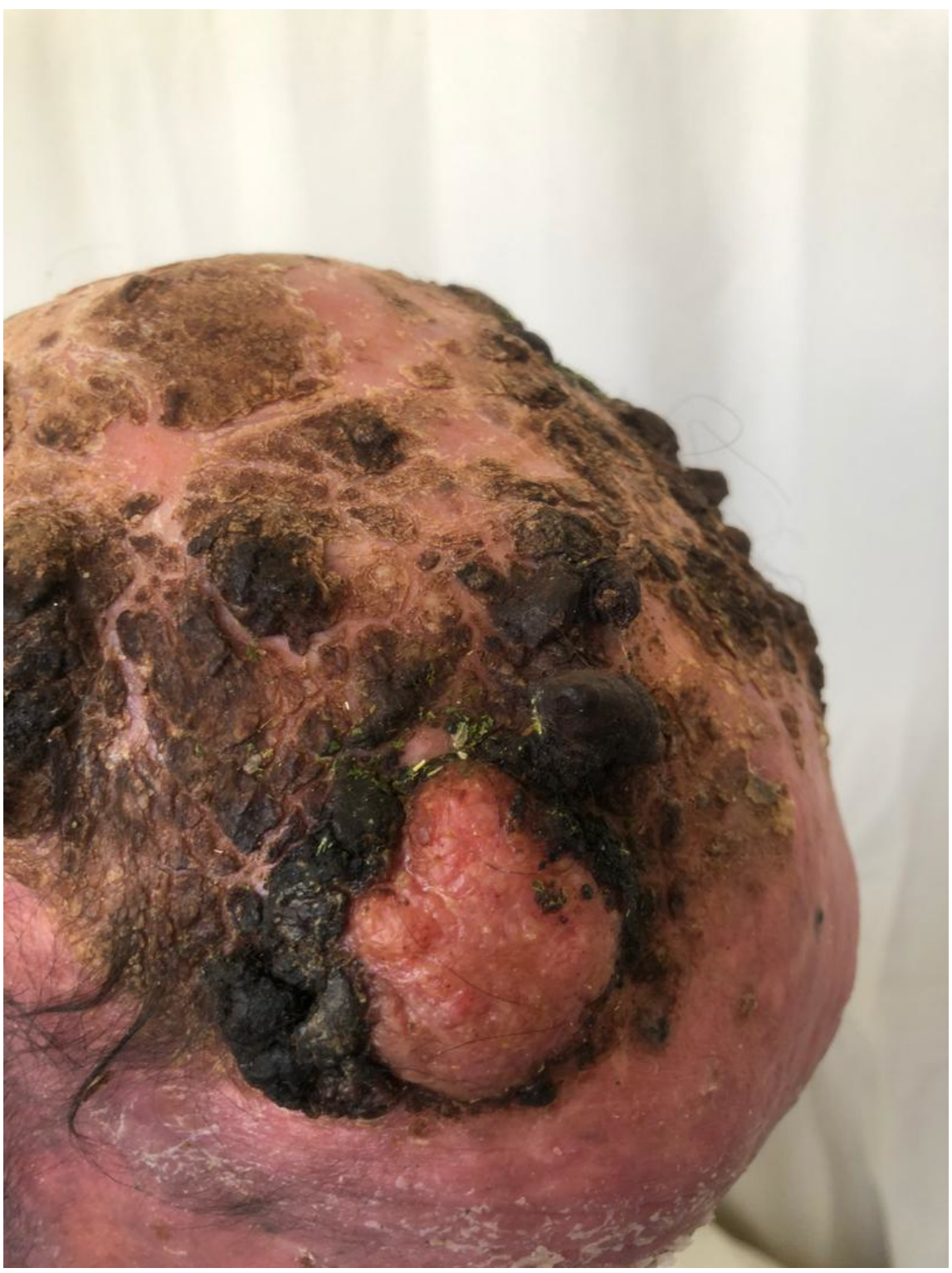

Figure 3: Multiple tumoral lesions present over the scalp including a $6 \times 5 \mathrm{~cm}$ erythematous, firm and adherent lesion over the frontal part. 


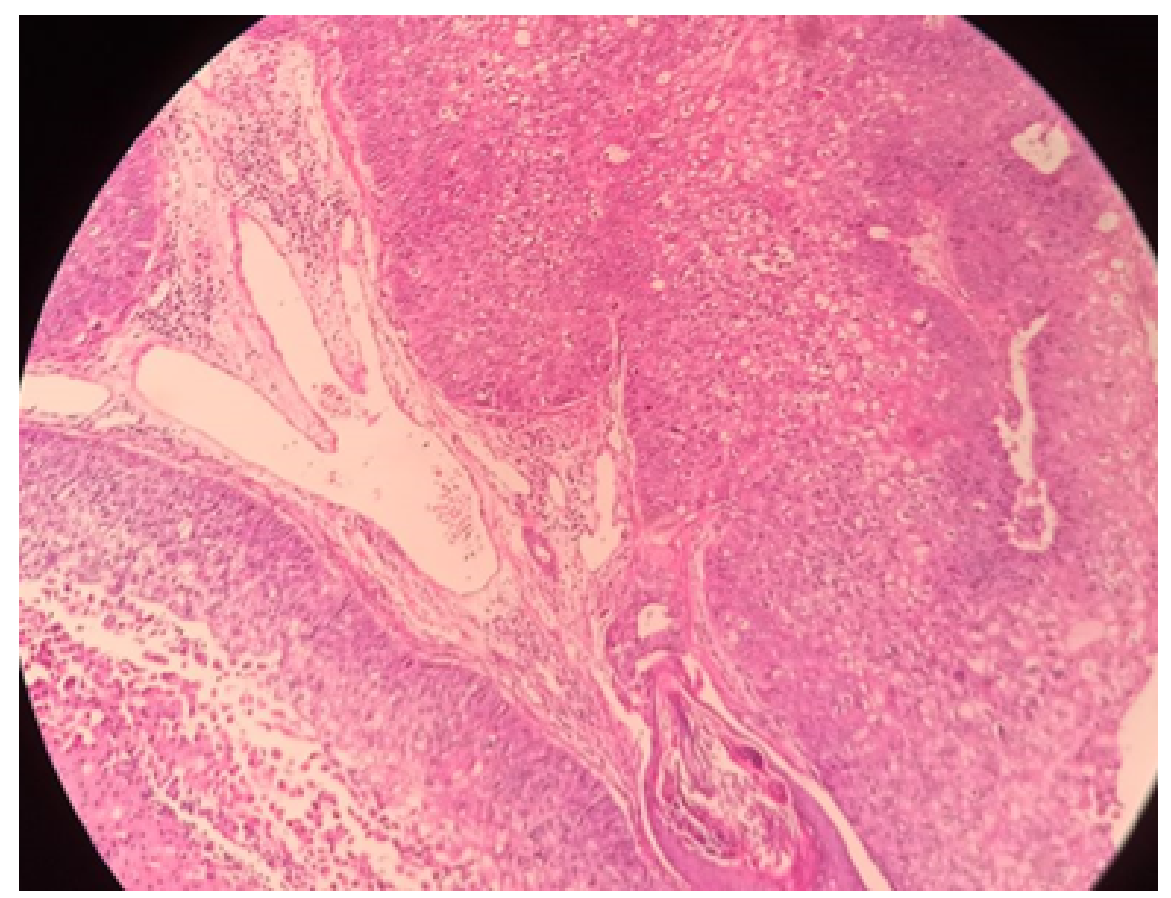

Figure 4: Histopathological schema showing neoplastic proliferation of epithelial cells with infiltration of lymphocytes. 


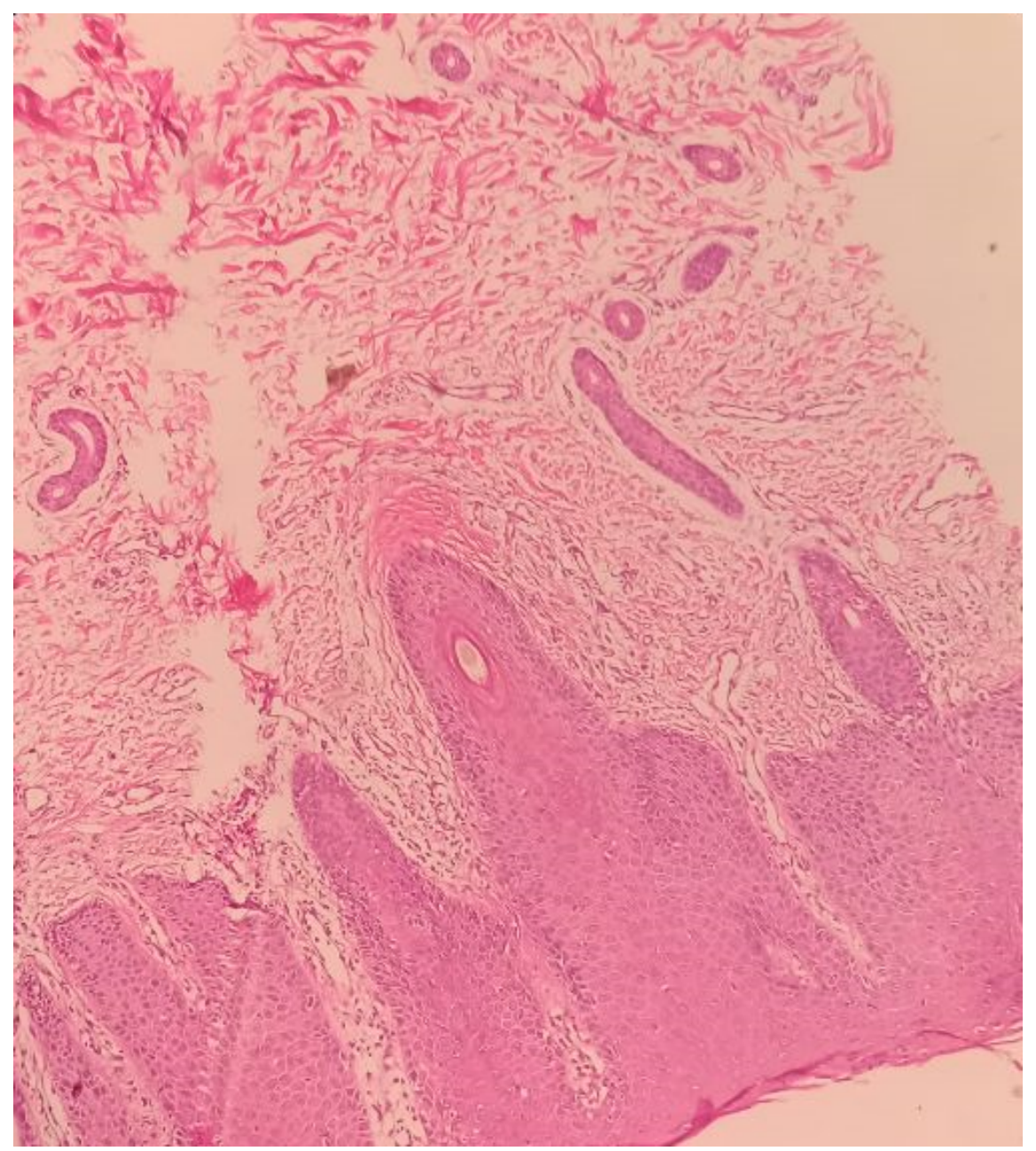

Figure 5: Histopathological schema showing parakeratosis and neutrophilic infiltration in parakeratotic layer (Monro microabscess) and downward elongation of rete ridges. Hypogranulosis, suprapillary thinning accompanied by dilated vessels, perivascular lymphocytic and a few neutrophilic infilteration are also seen, suggestive of psoriasis.

\section{Hosted file}

Figures.pdf available at https://authorea.com/users/383539/articles/499416-development-ofsquamous-cell-carcinoma-in-an-untreated-patient-of-psoriasis-a-case-report 